\title{
A Semisynthetic Fluorescent Sensor Protein for Glutamate
}

\author{
Matthias A. Brun, Kui-Thong Tan, ${ }^{\dagger}$ Rudolf Griss, Anna Kielkowska, Luc Reymond, and Kai Johnsson* \\ Institute of Chemical Sciences and Engineering, Institute of Bioengineering, National Centre of Competence in Research Chemical \\ Biology, École Polytechnique Fédérale de Lausanne (EPFL), Lausanne, Switzerland
}

\section{Supporting Information}

ABSTRACT: We report the semisynthesis of a fluorescent glutamate sensor protein on cell surfaces. Sensor excitation at $547 \mathrm{~nm}$ yields a glutamate-dependent emission spectrum between 550 and $700 \mathrm{~nm}$ that can be exploited for ratiometric sensing. On cells, the sensor displays a ratiometric change of 1.56 . The high sensitivity toward glutamate concentration changes of the sensor and its exclusive extracellular localization make it an attractive tool for glutamate sensing in neurobiology.

$\mathrm{T}$ he amino acid glutamate is the prevalent neurotransmitter in the vertebrate nervous system. It is used at well over $90 \%$ of the synapses in the human brain and influences essentially all forms of behavior, including consciousness, sensory perception, motor control, and mood. ${ }^{1}$ Further, glutamate is involved at most synapses that are modifiable, that is, that are capable of adapting to changing patterns of stimuli by enhancing or reducing the efficiency of synaptic transmission. ${ }^{2}$ These processes are thought to be responsible for high-order brain functions, such as learning and memory. Three fluorescent sensor proteins for investigating the role of glutamate in neurobiology have been developed so far. ${ }^{3-5}$ However, the modest performance of these sensor proteins has limited their use. Here we present a semisynthetic fluorescent sensor protein for glutamate which shows higher sensitivity toward glutamate concentration changes and operates at longer wavelengths than the previously reported sensors.

Semisynthetic fluorescent sensor proteins (Snifits), ${ }^{6,7}$ are fusion proteins consisting of SNAP-tag, ${ }^{8}$ CLIP-tag, ${ }^{9}$ and an analyte-binding protein (Figure 1A). SNAP- and CLIP-tag are labeled with a synthetic fluorescent ligand and a second synthetic fluorophore, respectively. The ligand binds to the binding protein in an intramolecular fashion and thereby keeps the sensor protein in a closed conformation. Free analyte can compete for binding to the binding protein and can shift the equilibrium to the open conformation. This shift can be detected by a change in the Förster resonance energy transfer (FRET) efficiency between the two fluorophores.

For the construction of a glutamate sensor protein based on the Snifit sensor concept, we have chosen the ionotropic glutamate receptor 5 (iGluR5) as the binding protein for two reasons. First, due to the modular construction of ionotropic glutamate receptors, it is possible to express its glutamate binding domain S1S2 as a soluble protein in bacteria while conserving both the high affinity and specificity toward glutamate. ${ }^{10,11}$ The possibility to characterize the soluble binding protein in vitro before its use on cell surfaces facilitates
A
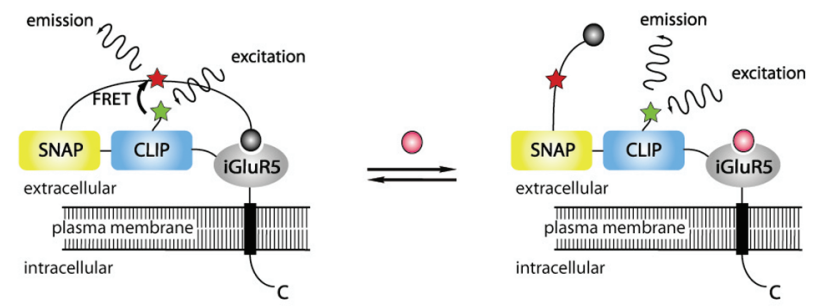

B

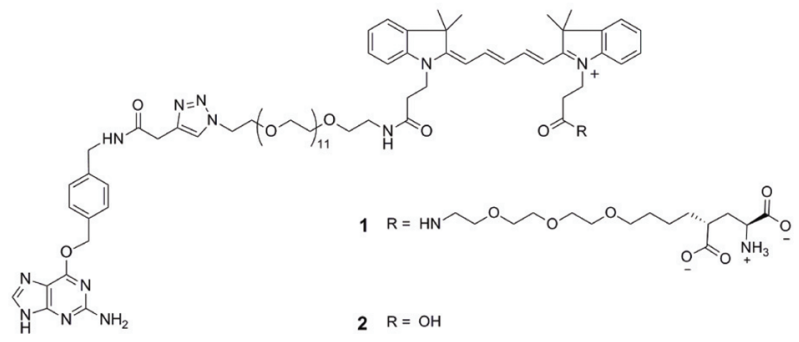

Figure 1. A Snifit for glutamate. (A) Design principle of the semisynthetic glutamate sensor protein. The protein part of the sensor is a fusion protein of SNAP-tag, CLIP-tag, and the glutamate binding protein iGluR5-S1S2 (iGluR5). The active semisynthetic sensor protein is obtained by labeling SNAP-tag with a molecule containing a Cy5 fluorophore (red star) and a terminal tethered glutamate analogue (gray ball), and by labeling CLIP-tag with a DY-547 fluorophore (green star). Glutamate (pink ball) at appropriate concentrations displaces the intramolecular ligand. (B) Structure of BG-PEG ${ }_{11}$-Cy5-glutamate $\mathbf{1}$ and the corresponding control molecule $\mathbf{2}$ for sensor protein labeling.

sensor development. Second, it is known that the stereoselective functionalization of the $\gamma$-carbon of the glutamate side chain does not significantly perturb its affinity toward iGluR5, ${ }^{12,13}$ suggesting an attachment point for the required synthetic tether. We therefore prepared the tethered glutamate analogue 1 (Figure 1B, Scheme S1-S3) that contains a Cy5 fluorophore and an $\mathrm{O}^{6}$-benzylguanine $(\mathrm{BG})$ group for reaction with SNAP-tag. To confirm that our tethered glutamate derivative 1 binds to $S 1 S 2$, we prepared a derivative without BG (Figure S1A) and tested its binding to purified recombinant S1S2 of iGluR5 by using fluorescence polarization (Figure S2). Assuming a similar effective molarity of the intramolecular ligand as in our previous work, ${ }^{6,7}$ the measured $K_{d}$ value of $7 \pm$ $1 \mu \mathrm{M}$ should enable the tethered glutamate 1 to keep the sensor in a closed confirmation in the absence of glutamate.

Next, we constructed the glutamate sensor protein SNAP_PP15_CLIP_iGluR5-S1S2 (abbreviated as Snifit-

Received: January 9, 2012

Published: April 23, 2012 
iGluR5). Snifit-iGluR5 is a linear fusion protein of SNAP-tag, CLIP-tag, and the glutamate binding protein iGluR5-S1S2, with a polyproline linker inserted between SNAP-tag and CLIP-tag. We have shown in our previous work that the inclusion of a rigid polyproline linker between SNAP- and CLIP-tag leads to greatly improved sensor proteins in terms of their maximum ratio changes. ${ }^{7}$ The polyproline linker increases the distance between the two fluorophores in the open state but not in the closed state of the sensor protein, which leads to a net increase of the sensor's dynamic range. ${ }^{7}$ The fusion protein was successfully expressed and purified from Escherichia coli Rosetta gami and labeled with the molecules BG-PEG 11 -Cy5-glutamate 1 and CLIP-Surface 547 (Figure S1B), which is a substrate for labeling CLIP-tag with the fluorescent dye DY-547 (Figure S3). We found that the addition of $1 \mathrm{mM}$ glutamate led to an increase of fluorescence emission of DY-547 and to a decrease of $\mathrm{Cy} 5$ fluorescence, indicating decreased FRET efficiency (Figure 2A). This behavior of Snifit-iGluR5 confirms the
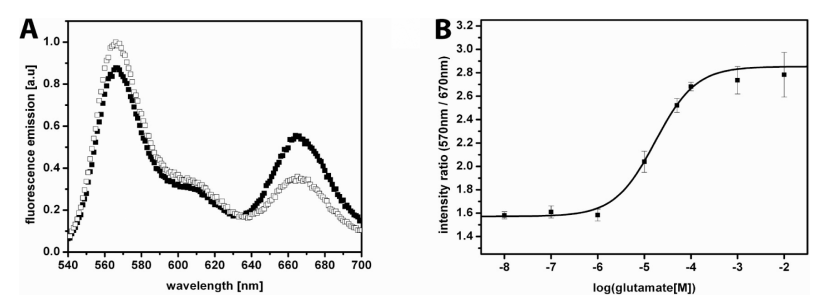

Figure 2. Snifit-iGluR5 as a sensor protein for glutamate. (A) Emission spectra of Snifit-iGluR5 at low and high concentrations of glutamate (10 nM, black; $10 \mathrm{mM}$, white). The addition of glutamate leads to an increase in the DY-547/Cy5 emission ratio with a maximum ratio change of $1.93 \pm 0.11$. (B) Fluorescence titration curve of SnifitiGluR5. Shown is the ratio of fluorescence donor $(570 \mathrm{~nm})$ and acceptor emission $(670 \mathrm{~nm})$ obtained by titrating the glutamate sensor protein with glutamate. The data are represented as the mean \pm standard deviation of triplicates. Data are fitted according to a singlesite binding isotherm.

general validity of our sensor concept. ${ }^{7}$ The maximum ratio change $\Delta R_{\max }$ of Snifit-iGluR5 amounts to $1.93 \pm 0.11$. By fitting the glutamate titration data to a single binding isotherm, we obtained a $K_{\mathrm{d}}^{\text {comp,glutamate }}=12 \pm 6 \mu \mathrm{M}$ (Figure $2 \mathrm{~B}$ ). SnifitiGluR5 labeled with BG-PEG 11 -Cy5 2 and CLIP-Surface 547 did not show any ratio change upon addition of glutamate (Figure S4, Scheme S4), supporting the hypothesis that the ratio change in Snifit-iGluR5 labeled with $\mathbf{1}$ is due to a specific displacement of the intramolecular ligand by free glutamate.

Snifit sensor proteins are very variable in terms of the employed fluorophore. ${ }^{6,7}$ To prove this versatility also for our Snifit-iGluR5, we labeled the sensor protein with BG-PEG ${ }_{11^{-}}$ Cy5-glutamate 1 and with BC-Cy7 (Figure S1D) which is a substrate for labeling CLIP-tag with the near-infrared dye Cy7. The combination of the two dyes Cy5 and Cy7 also led to a functional sensor protein, albeit with a slightly decreased $\Delta R_{\max }$ of $1.37 \pm 0.04$ Figure S5). For this reason, and since microscopes equipped with $\mathrm{Cy} 7$ filters are not very common, we decided to use the DY-547/Cy5 FRET pair for all further experiments.

We then performed the semisynthesis of our Snifit-iGluR5 on the surface of mammalian cells. To this end, we linked Snifit-iGluR5 to a truncated PDGF receptor (pDisplay) that then displays the sensor on the extracellular surface. The labeling of HEK 293T cells expressing Snifit-iGluR5 with SNAP-Surface $647(2 \mu \mathrm{M})$ and CLIP-Surface $547(10 \mu \mathrm{M})$ confirmed the presence of the sensor protein on the cell surface (Figure 3). The use of membrane impermeable dyes results in
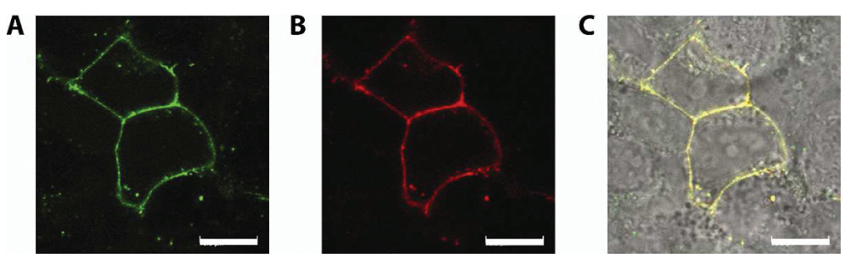

Figure 3. Fluorescence labeling of Snifit-iGluR5 on HEK 293T cells. SNAP PP15 CLIP iGluR5-S1S2 was labeled on the cell surface of HEK 293 T cells with the dyes DY-547 (A) and DY-647 (B) via their corresponding $\mathrm{O}^{2}$-benzylcytosine- $(\mathrm{BC})$ and BG-derivatives (Figure S1). (C) Overlay of DY-547 channel, DY-647 channel, and transmission channel. The white scale bar corresponds to $20 \mu \mathrm{m}$. Images were taken using a Leica SP5 WLL confocal microscope.

an exclusive labeling of Snifit-iGlurR5 on the cell surface, which should facilitate the specific sensing of secreted neurotransmitters.

Next, we investigated the response of Snifit-iGluR5 labeled with BG-PEG 11 -Cy5-glutamate 1 and with CLIP-Surface 547 upon perfusion of the cells with $1 \mathrm{mM}$ glutamate and found a reversible $\Delta R_{\max }$ of $1.56 \pm 0.05$ (Figure 4A, Figure S6). This

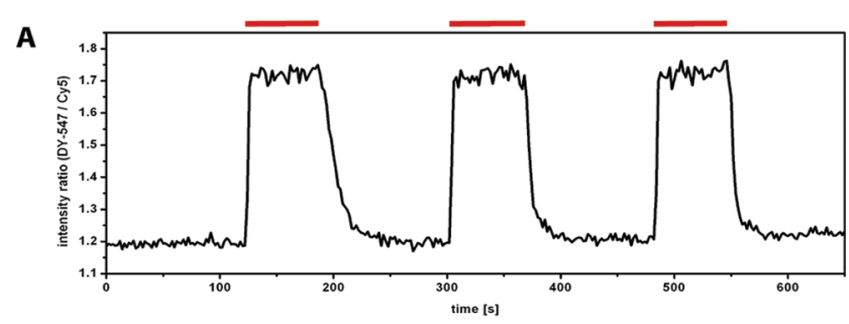

B
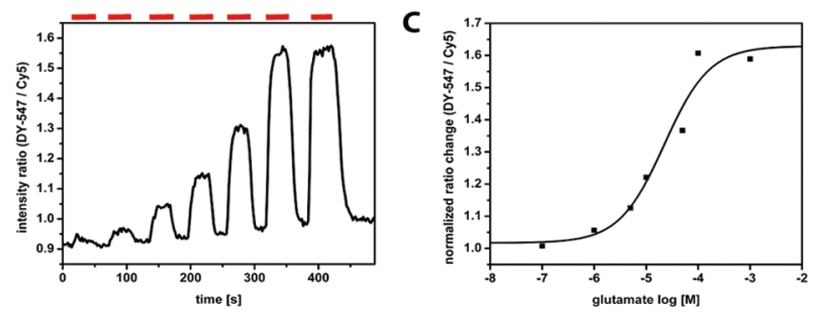

Figure 4. The sensor protein Snifit-iGluR5 on the surface of HEK 293T cells. (A) Time course of the intensity ratio of donor emission vs acceptor emission upon addition and removal of $1 \mathrm{mM}$ glutamate. The red bar indicates the time span of perfusion with glutamate. (B) Time course of perfusion of labeled Snifit-iGluR5 with increasing concentrations of glutamate. The red bar indicates the time span of perfusion with glutamate. Concentrations from left to right: $100 \mathrm{nM}, 1$ $\mu \mathrm{M}, 5 \mu \mathrm{M}, 10 \mu \mathrm{M}, 50 \mu \mathrm{M}, 100 \mu \mathrm{M}, 1 \mathrm{mM}$. (C) Glutamate titration curve of Snifit-iGluR5 on the extracellular surface of HEK 293T cells.

value is more than $20 \%$ larger than those reported for the previously described genetically encoded glutamate sensor proteins. ${ }^{3-5}$ In contrast, control experiments using the sensor protein labeled with BG-PEG $11-\mathrm{Cy} 5$ and CLIP-Surface 547 did not result in any intensity ratio change (Figure $\mathrm{S} 7$ ). We then also determined the $\Delta R_{\max }$ for cells expressing the sensor protein at different densities on their cell surface in order to investigate whether the $\Delta R_{\max }$ would depend on the expression level of the sensor protein. We found that the $\Delta R_{\max }$ remains constant for a wide range of different expression levels (Figure S8A). Only for cells that express the sensor protein at a level 
that approaches the detection limit, a decreased $\Delta R_{\max }$ was observed (Figure $\mathrm{S} 8 \mathrm{~A}$ ). The reduced $\Delta R_{\max }$ at very low expression levels can be explained by the increasing relative contribution of background fluorescence. Nevertheless, our analysis shows that the Snifit-iGluR5 also performs reliably even at the detection limit of the used fluorophores. This property of our sensor protein might be advantageous in experimental settings where a high expression level of sensor protein is problematic.

To test the selectivity of Snifit-iGluR5 for glutamate, we measured the response of our sensor protein toward other physiologically relevant amino acids and neurotransmitters. Sequential perfusion of cells expressing Snifit-iGluR5 on the cell surface with aspartate, GABA, glycine, and glutamate only resulted in a detectable ratio change when cells were perfused with glutamate, demonstrating the specificity of the sensor (Figure S9).

We then determined the affinity of our Snifit for glutamate in single cell measurements and found a $K_{d}^{\text {comp,glutamate }}$ of $15 \pm 5$ $\mu \mathrm{M}$ (Figure 4B,C).

As in our previous study, ${ }^{7}$ the $K_{d}{ }^{\text {comp,glutamate }}$ does not change for different cells expressing the sensor protein at varying concentrations on the cell surface (Figure S8B). The biologically relevant glutamate concentrations can vary significantly in the neurological system. For example, the glutamate concentration in the synaptic cleft lies at the high nanomolar level at resting state, but reaches a very short-lived millimolar concentration burst following an action potential. ${ }^{14}$ Around astrocytes, the extracellular glutamate concentration is estimated to be around $5 \mu \mathrm{M} .^{15}$ Accordingly, the measured $K_{\mathrm{d}}^{\text {comp,glutamate }}$ of $15 \mu \mathrm{M}$ for our Snifit-iGluR5 on cells is in an appropriate range to study glutamate transients in neurological systems.

Another critical parameter of a glutamate sensor is its temporal resolution. The published activation and deactivation rates of iGluR5 upon binding and unbinding of glutamate are in the millisecond range and similar binding and unbinding rates could be expected for Snifit-iGluR5. ${ }^{16}$ In our perfusion experiments (Figure 4), the sensor closes and opens within seconds. However, these rates reflect the performance of our perfusion system which only allows the exchange of the bath solution within 3 to $4 \mathrm{~s}$ (Figure S10). A detailed analysis of the kinetics of our glutamate sensor will require a different experimental setup and will need to be investigated in future experiments.

In conclusion, we have developed a novel semisynthetic sensor protein for glutamate based on our recently developed Snifit sensor concept. The observed maximum ratio change for Snifit-iGluR5 on cell surfaces is higher than that of any other existing glutamate biosensor. Further, the exclusive labeling of Snifit-iGluR5 on the cell surface with far-red fluorophores should facilitate applications on tissue samples or in vivo as well as its use in combination with other biosensors (multiplexing). These properties should make Snifit-iGluR5 a very useful tool to study the glutamate dynamics in the nervous system.

\section{ASSOCIATED CONTENT}

\section{S Supporting Information}

Synthetic procedures, characterizations of the Snifit-iGluR5 in vitro and on the cell surface; additional figures. This material is available free of charge via the Internet at http://pubs.acs.org.

\section{AUTHOR INFORMATION}

\section{Corresponding Author}

kai.johnsson@epfl.ch

Present Address

${ }^{\dagger}$ Department of Chemistry, National Tsing Hua University, Hsinchu 30013, Taiwan.

\section{Notes}

The authors declare no competing financial interest.

\section{ACKNOWLEDGMENTS}

This work was supported by funding from the Swiss National Science Foundation and EPFL. The authors thank Dr. M. L. Mayer for kindly providing us with the plasmid encoding the iGluR5-S1S2 sequence and A. Masharina for technical assistance.

\section{REFERENCES}

(1) Watkins, J. C.; Evans, R. H. Annu. Rev. Pharmacol. Toxicol. 1981, 21, 165-204.

(2) Kandel, E. R. Science 2001, 294 (5544), 1030-1038.

(3) Okumoto, S.; Looger, L.; Micheva, K.; Reimer, R.; Smith, S.; Frommer, W. Proc. Natl. Acad. Sci. U.S.A. 2005, 102 (24), 8740-8745.

(4) Hires, S.; Zhu, Y.; Tsien, R. Proc. Natl. Acad. Sci. U.S.A. 2008, 105 (11), 4411-4416.

(5) Hirose, K.; Namiki, S.; Sakamoto, H.; Iinuma, S.; Iino, M. Eur. J. Neurosci. 2007, 25 (8), 2249-2259.

(6) Brun, M. A.; Tan, K. T.; Nakata, E.; Hinner, M. J.; Johnsson, K. J. Am. Chem. Soc. 2009, 131 (16), 5873-5884.

(7) Brun, M. A.; Griss, R.; Reymond, L.; Tan, K. T.; Piguet, J.; Peters, R. J.; Vogel, H.; Johnsson, K. J. Am. Chem. Soc. 2011, 133 (40), 16235-42.

(8) Keppler, A.; Gendreizig, S.; Gronemeyer, T.; Pick, H.; Vogel, H.; Johnsson, K. Nat. Biotechnol. 2003, 21 (1), 86-89.

(9) Gautier, A.; Juillerat, A.; Heinis, C.; Correa, I.; Kindermann, M.; Beaufils, F.; Johnsson, K. Chem. Biol. 2008, 15 (2), 128-136.

(10) Kuusinen, A.; Arvola, M.; Keinanen, K. EMBO J. 1995, 14 (24), 6327-6332.

(11) Chen, G. Q.; Gouaux, E. Pro. Natl. Acad. Sci. U.S.A. 1997, 94 (25), 13431-13436.

(12) Volgraf, M.; Gorostiza, P.; Numano, R.; Kramer, R.; Isacoff, E.; Trauner, D. Nat. Chem. Biol. 2006, 2 (1), 47-52.

(13) Volgraf, M.; Gorostiza, P.; Szobota, S.; Helix, M. R.; Isacoff, E. Y.; Trauner, D. J. Am. Chem. Soc. 2007, 129 (2), 260-261.

(14) Bergles, D. E.; Diamond, J. S.; Jahr, C. E. Curr. Opin. Neurbiol. 1999, 9 (3), 293-8.

(15) McLamore, E. S.; Mohanty, S.; Shi, J.; Claussen, J.; Jedlicka, S. S.; Rickus, J. L.; Porterfield, D. M. J. Neurosci. Methods 2010, 189 (1), $14-22$.

(16) Dingledine, R.; Borges, K.; Bowie, D.; Traynelis, S. F. Pharmacol. Rev. 1999, 51 (1), 7-61. 